REF. TUW 99-07

\title{
Generalized 2D BF Model quantized in the axial gauge
}

\author{
R. Leitgeb ${ }^{1}$, J. Rant ${ }^{1}$, M. Schweda and H. Zerrouki \\ Institut für Theoretische Physik, Technische Universität Wien \\ Wiedner Hauptstraße 8-10, A-1040 Wien, Austria
}

\begin{abstract}
We discuss the ultraviolet finiteness of the two-dimensional BF model coupled to topological matter quantized in the axial gauge. This noncovariant gauge fixing avoids the infrared problem in the two-dimensional space-time. The BF model together with the matter coupling is obtained by dimensional reduction of the ordinary three-dimensional BF model. This procedure furnishes the usual linear vector supersymmetry and an additional scalar supersymmetry. The whole symmetry content of the model allows to apply the standard algebraic renormalization procedure which we use to prove that this model is ultraviolet finite and anomaly free to all orders of perturbation theory.
\end{abstract}

\footnotetext{
${ }^{1}$ Work supported in part by the "Fonds zur Förderung der Wissenschaflicher Forschung", under Project Grant Number P11354-PHY.
} 


\section{Introduction}

Topological field models have been an object of intense interest over the last decade and brought significant developments to the understanding of the topology and geometry of low dimensional manifolds [1, 2]. The main property of the topological models [3] is the fact that the observables depend only on the global structure of the space-time manifold on which they are defined, e.g. no physical degrees of freedom exist locally. In particular, they are independent of any metric which may be used to define the classical theory.

There are two different types of topological field theories. The first one is called Wittentype [4] whose whole gauge fixed action may be written as a total BRS variation. The most prominent example of Witten-type models is the topological Yang-Mills theory. The second type of topological models are the Schwarz-type models characterized by the fact that only the gauge fixed part of the action is an exact BRS-variation. The examples of the Schwarz-type models are Chern-Simons and BF theories. A common feature of such models is the presence of the so-called topological linear vector supersymmetry. The corresponding operator $\delta_{\mu}$ and the usual BRS-operator $s$ form a graded algebra of WessZumino type:

$$
\left\{s, \delta_{\mu}\right\}=\partial_{\mu} .
$$

The aim of the present work is to analyze the ultraviolet behavior of two-dimensional $\mathrm{BF}$ model with a matter coupling [5] quantized in the axial gauge. The infrared and ultraviolet behavior of the pure two-dimensional BF model has been already discussed in [6, ]]. Usually the propagators in two space-time dimensions are not well-defined in the infrared region. In the present work, however, the use of the axial gauge removes the singular behavior at long distances. To carry out the proof of perturbative finiteness we will use the ordinary algebraic renarmalization procedure in the context of BRS-symmetry [8, 9, 15]. Besides the usual BRS-symmetry there exists also the topological linear vector supersymmetry [9] together with an additional topological scalar supersymmetry. The latter scalar supersymmetry is a by-product in reducing the three-dimensional BF model to two-dimensional space-time. The whole symmetry content of the model is basis for the proof of the ultraviolet finiteness.

The paper is organized as follows. In Section 2 we present the classical algebraic properties of the model at the classical level. We display the BRS-transformations, the topological linear vector supersymmetry and the additional scalar supersymmetry. In Section 3 we discuss the proof of perturbative finiteness of the model by analyzing its stability. The same arguments as in [13] imply that the model is free of anomalies. 


\section{The classical model}

The action of classical BF model living on manifolds $\mathcal{M}$ with $(n+2)$-dimensions can be defined [1, 3, 9] according to

$$
S_{B F}=\operatorname{Tr} \int_{\mathcal{M}} B F=\frac{1}{2 n !} \operatorname{Tr} \int_{\mathcal{M}} d^{n+2} x \varepsilon^{\mu_{1} \ldots \mu_{n+2}} B_{\mu_{1} \ldots \mu_{n}} F_{\mu_{n+1} \mu_{n+2}},
$$

where $F$ is a two-form

$$
F=d A+\frac{1}{2}[A, A]=\frac{1}{2} F_{\mu \nu} d x^{\mu} d x^{\nu},
$$

$\mathrm{A}$ is the usual gauge connection one-form and $\mathrm{B}$ is a $\mathrm{n}$-form:

$$
B=\frac{1}{n !} B_{\mu_{1} \ldots \mu_{n}} d x^{\mu_{1}} \ldots d x^{\mu_{n}}
$$

This action has a topological character since it is independent of the metric of the manifold $\mathcal{M}$.

In the case of two-dimensional flat Euclidean space-time the action of the BF model reads

$$
S_{\text {inv }}^{(1)}=\frac{1}{2} \int_{\mathcal{M}} d^{2} x \varepsilon^{\mu \nu} F_{\mu \nu}^{a} \phi^{a}
$$

where $\varepsilon^{\mu \nu}$ is the totally antisymmetric Levi-Civita tensor (with $\varepsilon^{12}=+1$ ), $\phi^{a}$ is a scalar field and $F_{\mu \nu}^{a}$ is the field strength given by

$$
F_{\mu \nu}^{a}=\partial_{\mu} A_{\nu}^{a}-\partial_{\nu} A_{\mu}^{a}+f^{a b c} A_{\mu}^{b} A_{\nu}^{c}
$$

Here, $A_{\mu}^{a}$ is the gauge field with the group index $a$. All fields belong to the adjoint representation of some compact semi-simple gauge group $G$ whose structure constants $f^{a b c}$ are completely antisymmetric in their indices. The generators of the Lie algebra are chosen to be anti-hermitian and subject to $\left[T^{a}, T^{b}\right]=f^{a b c} T^{c}$ and $\operatorname{Tr}\left(T^{a} T^{b}\right)=\delta^{a b}$.

The action (2.4) is invariant under the following infinitesimal gauge symmetry

$$
\begin{aligned}
\delta^{(1)} A_{\mu}^{a} & =\partial_{\mu} \theta^{a}+f^{a b c} A_{\mu}^{b} \theta^{c} \equiv\left(D_{\mu} \theta\right)^{a}, \\
\delta^{(1)} \phi^{a} & =-f^{a b c} \theta^{b} \phi^{c},
\end{aligned}
$$

where $\theta^{a}$ and $D_{\mu}$ stand for a local gauge parameter and the covariant derivative respectively. This action has much been investigated in [6, 0]. Following [5, 13] we enlarge the model by adding to (2.4) the following topological matter interaction term:

$$
S_{i n v}^{(2)}=\int_{\mathcal{M}} d^{2} x \varepsilon^{\mu \nu}\left(D_{\mu} B_{\nu}\right)^{a} X^{a},
$$

with two additional fields: a vector field $B_{\mu}^{a}$ and a scalar field $X^{a}$. The action (2.7) is invariant under $\delta^{(1)}$ :

$$
\begin{aligned}
\delta^{(1)} B_{\mu}^{a} & =-f^{a b c} \theta^{b} B_{\mu}^{c} \\
\delta^{(1)} X^{a} & =-f^{a b c} \theta^{b} X^{c}
\end{aligned}
$$


The introduction of the matter coupling (2.7) implies that the total action

$$
S_{i n v}=S_{i n v}^{(1)}+S_{i n v}^{(2)}
$$

possesses an additional gauge symmetry given by

$$
\begin{aligned}
\delta^{(2)} A_{\mu}^{a} & =0, \\
\delta^{(2)} \phi^{a} & =-f^{a b c} \Theta^{b} X^{c}, \\
\delta^{(2)} X^{a} & =0 \\
\delta^{(2)} B_{\mu}^{a} & =\left(D_{\mu} \Theta\right)^{a},
\end{aligned}
$$

where $\Theta^{a}$ is another infinitesimal local parameter.

As usual, the quantization of gauge field models requires a gauge fixing in order to guarantee the existance of the gauge field propagators. This is done consistently by the introduction of the Faddeev-Popov ghost fields in the context of the BRS-framework [8]. Since there are two different gauge symmetries in the model, the BRS-quantization procedure requires two sets of ghost fields with the corresponding Lagrange multiplier fields. We therefore introduce two Faddeev-Popov ghosts $\left(c^{a}, \lambda^{a}\right)$ with the corresponding antighosts $\left(\bar{c}^{a}, \bar{\lambda}^{a}\right)$ and two Lagrange multipliers $\left(b^{a}, d^{a}\right)$. We choose the axial gauge and add the following gauge fixing term to the action $S_{\text {inv }}$ :

$$
\begin{aligned}
S_{g f} & =s \int_{\mathcal{M}} d^{2} x\left(\bar{c}^{a} n^{\mu} A_{\mu}^{a}+\bar{\lambda}^{a} n^{\mu} B_{\mu}^{a}\right) \\
& =\int_{\mathcal{M}} d^{2} x\left(b^{a} n^{\mu} A_{\mu}^{a}+d^{a} n^{\mu} B_{\mu}^{a}-\bar{c}^{a} n^{\mu}\left(D_{\mu} c\right)^{a}-\bar{\lambda}^{a} n^{\mu}\left(D_{\mu} \lambda\right)^{a}+f^{a b c} \bar{\lambda}^{a} c^{b} n^{\mu} B_{\mu}^{c}\right)
\end{aligned}
$$

where $n^{\mu}$ is a fixed gauge direction. The complete gauge fixed action is, by construction, BRS-invariant

$$
s\left(S_{i n v}+S_{g f}\right)=0,
$$

where the nilpotent and nonlinear BRS-transformation read as

$$
\begin{aligned}
s A_{\mu}^{a} & =\left(D_{\mu} c\right)^{a}, \\
s B_{\mu}^{a} & =\left(D_{\mu} \lambda\right)^{a}-f^{a b c} c^{b} B_{\mu}^{c}, \\
s \phi^{a} & =-f^{a b c} c^{b} \phi^{c}-f^{a b c} \lambda^{b} X^{c} \\
s X^{a} & =-f^{a b c} c^{b} X^{c}, \\
s c^{a} & =-\frac{1}{2} f^{a b c} c^{b} c^{c} \\
s \lambda^{a} & =-f^{a b c} c^{b} \lambda^{c}, \\
s \bar{c}^{a} & =b^{a}, \quad s b^{a}=0 \\
s \bar{\lambda}^{a} & =d^{a}, \quad s d^{a}=0 \\
s^{2} & =0 .
\end{aligned}
$$


In order to control the $n^{\mu}$-dependance of the theory one enlarges the BRS-transformations by allowing also a variation of the axial vector $n^{\mu}$ [10]:

$$
s n^{\mu}=\chi^{\mu}, \quad s \chi^{\mu}=0,
$$

and by adding the following term to the action

$$
S_{n}=-\operatorname{Tr} \int_{\mathcal{M}} d^{2} x\left(\bar{c}^{a} \chi^{\mu} A_{\mu}^{a}+\bar{\lambda}^{a} \chi^{\mu} B_{\mu}^{a}\right)
$$

Here, $\chi^{\mu}$ is an anticommuting parameter. Obviously, the new action

$$
S=S_{i n v}+S_{g f}+S_{n}
$$

is now invariant under the enlarged BRS-transformations (2.13) together with (2.14). The physical situation is represented by putting $\chi^{\mu}$ to zero. We present the canonical dimensions and the Faddeev-Popov charges of all fields in Table 1.

\begin{tabular}{|c|c|c|c|c|c|c|c|c|c|c|c|c|}
\hline & $A_{\mu}^{a}$ & $B_{\mu}^{a}$ & $\phi^{a}$ & $X^{a}$ & $c^{a}$ & $\lambda^{a}$ & $\bar{c}^{a}$ & $\lambda^{a}$ & $b^{a}$ & $d^{a}$ & $n_{\mu}$ & $\chi^{\mu}$ \\
\hline $\operatorname{dim}$ & 1 & 1 & 0 & 0 & 0 & 0 & 1 & 1 & 1 & 1 & 0 & 0 \\
\hline$\phi \pi$ & 0 & 0 & 0 & 0 & 1 & 1 & -1 & -1 & 0 & 0 & 0 & 1 \\
\hline
\end{tabular}

Table 1: Dimensions and Faddeev-Popov charges of the fields

As usual for topological field models the action (2.16) possesses besides the BRS-symmetry an invariance with respect to the linear vector supersymmetry:

$$
\begin{aligned}
& \delta_{\mu} A_{\mu}^{a}=0, \quad \delta_{\mu} B_{\mu}^{a}=0, \\
& \delta_{\mu} \phi^{a}=\varepsilon_{\mu \nu} n^{\nu} \bar{c}^{a} \quad, \quad \delta_{\mu} X^{a}=\varepsilon_{\mu \nu} n^{\nu} \bar{\lambda}^{a}, \\
& \delta_{\mu} c^{a}=A_{\mu}^{a}, \quad \delta_{\mu} \lambda^{a}=B_{\mu}^{a}, \\
& \delta_{\mu} \bar{c}^{a}=0, \quad \delta_{\mu} \bar{\lambda}^{a}=0, \\
& \delta_{\mu} b^{a}=\partial_{\mu} \bar{c}^{a}, \quad \delta_{\mu} d^{a}=\partial_{\mu} \bar{\lambda}^{a}, \\
& \delta_{\mu} n^{\nu}=0, \quad \delta_{\mu} \chi^{\nu}=0 .
\end{aligned}
$$

One can easily verify that

$$
\delta_{\mu} S=0
$$

Moreover, the action (2.16) is left invariant under a further topological scalar supersymmetric transformation acting on the fields as follows

$$
\begin{array}{ll}
\delta A_{\mu}^{a}=-\varepsilon_{\mu \nu} n^{\nu} \bar{\lambda}^{a}, & \delta B_{\mu}^{a}=-\varepsilon_{\mu \nu} n^{\nu} \bar{c}^{a}, \\
\delta \phi^{a}=0 & \delta X^{a}=0, \\
\delta c^{a}=X^{a}, & \delta \lambda^{a}=\phi^{a}, \\
\delta \bar{c}^{a}=0, & \delta \bar{\lambda}^{a}=0 \\
\delta b^{a}=0, & \delta d^{a}=0 \\
\delta n^{\mu}=0, & \delta \chi^{\mu}=0 .
\end{array}
$$


This symmetry! may be interpreted in the following manner. First note that twodimensional $\mathrm{BF}$ model coupled to topological matter can be obtained by a dimensional reduction of the three-dimensional BF model. The symmetry (2.19) corresponds then to the third component of the topological vector supersymmetry of the three-dimensional BF model [14]. The BRS-operator (2.13), the vector supersymmetry $\delta_{\mu}(2.17)$ and the operator $\delta$ defined in (2.19) form an algebra of Wess-Zumino type which closes on-shell on the translations:

$$
\begin{aligned}
\{s, s\} & =0 \\
\left\{s, \delta_{\mu}\right\} A_{\nu}^{a} & =\partial_{\mu} A_{\nu}^{a}+\varepsilon_{\mu \nu} \frac{\delta S}{\delta \phi^{a}}, \\
\left\{s, \delta_{\mu}\right\} \phi^{a} & =\partial_{\mu} \phi^{a}+\varepsilon_{\mu \nu} \frac{\delta S}{\delta A_{\nu}^{a}}, \\
\left\{s, \delta_{\mu}\right\} B_{\nu}^{a} & =\partial_{\mu} B_{\nu}^{a}+\varepsilon_{\mu \nu} \frac{\delta S}{\delta X^{a}}, \\
\left\{s, \delta_{\mu}\right\} X^{a} & =\partial_{\mu} X^{a}+\varepsilon_{\mu \nu} \frac{\delta S}{\delta B_{\nu}^{a}}, \\
\left\{s, \delta_{\mu}\right\} \psi^{a} & =\partial_{\mu} \psi^{a}, \forall \psi^{a} \in\left\{c^{a}, \lambda^{a}, \bar{c}^{a}, \bar{\lambda}^{a}, b^{a}, d^{a}\right\} \\
\{s, \delta\} A_{\mu}^{a} & =-\varepsilon_{\mu \nu} \frac{\delta S}{\delta B_{\nu}^{a}}, \\
\{s, \delta\} B_{\nu}^{a} & =-\varepsilon_{\mu \nu} \frac{\delta S}{\delta A_{\nu}^{a}}, \\
\{s, \delta\} \psi^{a} & =0, \forall \psi^{a} \in\left\{\phi^{a}, X^{a}, c^{a}, \lambda^{a}, \bar{c}^{a}, \bar{\lambda}^{a}, b^{a}, d^{a}\right\}
\end{aligned}
$$

Moreover, the following algebraic relations hold

$$
\begin{array}{r}
\left\{\delta_{\mu}, \delta_{\nu}\right\}=0 \\
\left\{\delta_{\mu}, \delta\right\}=0 \\
\{\delta, \delta\}=0
\end{array}
$$

In order to describe the BRS-symmetry content consistently at the functional level, we introduce a set of external sources coupled to the nonlinear BRS-variations of the quantum fields:

$$
S_{e x t}=\int_{\mathcal{M}} d^{2} x\left[\Omega^{\mu a}\left(s A_{\mu}^{a}\right)+L^{a}\left(s c^{a}\right)+\varrho^{a}\left(s \phi^{a}\right)+\sigma^{\mu a}\left(s B_{\mu}^{a}\right)+D^{a}\left(s \lambda^{a}\right)+Y^{a}\left(s X^{a}\right)\right] .
$$

We display the canonical dimensions and the Faddeev-Popov charges of the external sources in Table 1 .

\footnotetext{
${ }^{1}$ The use of this symmetry simplifies the the proof of the finiteness of the theory as we will see later on.
} 


\begin{tabular}{|c|c|c|c|c|c|c|}
\hline & $\Omega^{\mu a}$ & $L^{a}$ & $\varrho^{a}$ & $\sigma^{\mu a}$ & $D^{a}$ & $Y^{a}$ \\
\hline $\operatorname{dim}$ & 1 & 2 & 2 & 1 & 2 & 2 \\
\hline$\phi \pi$ & -1 & -2 & -1 & -1 & -2 & -1 \\
\hline
\end{tabular}

Table 2: Dimensions and Faddeev-Popov charges of the external sources

The complete action

$$
\Sigma^{(0)}=S_{i n v}+S_{g f}+S_{n}+S_{e x t}
$$

obeys the Slavnov identity:

$$
\begin{aligned}
\mathcal{S}\left(\Sigma^{(0)}\right) & =\int_{\mathcal{M}} d^{2} x\left[\frac{\delta \Sigma^{(0)}}{\delta \Omega^{\mu a}} \frac{\delta \Sigma^{(0)}}{\delta A_{\mu}^{a}}+\frac{\delta \Sigma^{(0)}}{\delta L^{a}} \frac{\delta \Sigma^{(0)}}{\delta c^{a}}+\frac{\delta \Sigma^{(0)}}{\delta \varrho^{a}} \frac{\delta \Sigma^{(0)}}{\delta \phi^{a}}+\frac{\delta \Sigma^{(0)}}{\delta \sigma^{\mu a}} \frac{\delta \Sigma^{(0)}}{\delta B_{\mu}^{a}}+\right. \\
& \left.+\frac{\delta \Sigma^{(0)}}{\delta D^{a}} \frac{\delta \Sigma^{(0)}}{\delta \lambda^{a}}+\frac{\delta \Sigma^{(0)}}{\delta Y^{a}} \frac{\delta \Sigma^{(0)}}{\delta X^{a}}+b^{a} \frac{\delta \Sigma^{(0)}}{\delta \bar{c}^{a}}+d^{a} \frac{\delta \Sigma^{(0)}}{\delta \bar{\lambda}^{a}}\right]+\chi^{\mu} \frac{\partial \Sigma^{(0)}}{\partial n^{\mu}}=0 .
\end{aligned}
$$

The introduction of external sources induces a modified Ward-operator for the linear vector supersymmetry $\mathcal{W}_{\mu}$ :

$$
\begin{aligned}
\mathcal{W}_{\mu} & =\int_{\mathcal{M}} d^{2} x\left[\varepsilon_{\mu \nu} \varrho^{a} \frac{\delta}{\delta A_{\nu}^{a}}+\varepsilon_{\mu \nu}\left(n^{\nu} \bar{c}^{a}-\Omega^{\nu a}\right) \frac{\delta}{\delta \phi^{a}}+A_{\mu}^{a} \frac{\delta}{\delta c^{a}}+\partial_{\mu} \bar{c}^{a} \frac{\delta}{\delta b^{a}}+L^{a} \frac{\delta}{\delta \Omega^{\mu a}}+\right. \\
& \left.+\varepsilon_{\mu \nu} Y^{a} \frac{\delta}{\delta B_{\nu}^{a}}+\varepsilon_{\mu \nu}\left(n^{\nu} \bar{\lambda}^{a}-\sigma^{\nu a}\right) \frac{\delta}{\delta X^{a}}+B_{\mu} \frac{\delta}{\delta \lambda^{a}}+\partial_{\mu} \bar{\lambda}^{a} \frac{\delta}{\delta d^{a}}+D^{a} \frac{\delta}{\delta \sigma^{\mu a}}\right],
\end{aligned}
$$

and the vector supersymmetry is broken linearly

$$
\mathcal{W}_{\mu} \Sigma^{(0)}=\Delta_{\mu}
$$

where

$$
\begin{aligned}
\Delta_{\mu} & =\int_{\mathcal{M}} d^{2} x\left[-\varrho^{a} \partial_{\mu} \phi^{a}+\varepsilon_{\mu \nu} n^{\nu} \varrho^{a} b^{a}+\varepsilon_{\mu \nu} n^{\nu} Y^{a} d^{a}-Y^{a} \partial_{\mu} X^{a}-\sigma^{\nu a} \partial_{\mu} B_{\nu}^{a}+\right. \\
& \left.+D^{a} \partial_{\mu} \lambda^{a}+L^{a} \partial_{\mu} c^{a}-\Omega^{\nu a} \partial_{\mu} A_{\nu}^{a}\right] .
\end{aligned}
$$

Note that the breaking (2.27) is linear in the quantum fields and therefore harmless at the quantum level. Moreover, the topological scalar supersymmetry is expressed by the following Ward-operator $\mathcal{D}$ :

$$
\begin{aligned}
\mathcal{D} & =\int_{\mathcal{M}} d^{2} x\left[\varepsilon_{\mu \nu}\left(\sigma^{\nu a}-n^{\nu} \bar{\lambda}^{a}\right) \frac{\delta}{\delta A_{\mu}^{a}}+\varepsilon_{\mu \nu}\left(\Omega^{\nu a}-n^{\nu} \bar{c}^{a}\right) \frac{\delta}{\delta B_{\mu}^{a}}+X^{a} \frac{\delta}{\delta c^{a}}+\right. \\
& \left.+\phi^{a} \frac{\delta}{\delta \lambda^{a}}+D^{a} \frac{\delta}{\delta \varrho^{a}}+L^{a} \frac{\delta}{\delta Y^{a}}\right],
\end{aligned}
$$

with

$$
\mathcal{D} \Sigma^{(0)}=\Delta,
$$


where the breaking

$$
\Delta=\int_{\mathcal{M}} d^{2} x \varepsilon_{\mu \nu} n^{\mu}\left(\sigma^{\nu a} b^{a}+\Omega^{\nu a} d^{a}\right)
$$

is linear in quantum fields, hence being harmless at the quantum level. For later use we introduce the linearized Slavnov operator $\mathcal{S}_{\Sigma}^{(0)}$ :

$$
\begin{aligned}
\mathcal{S}_{\Sigma^{(0)}} & =\int_{\mathcal{M}} d^{2} x\left[\frac{\delta \Sigma^{(0)}}{\delta \Omega^{\mu a}} \frac{\delta}{\delta A_{\mu}^{a}}+\frac{\delta \Sigma^{(0)}}{\delta A_{\mu}^{a}} \frac{\delta}{\delta \Omega^{\mu a}}+\frac{\delta \Sigma^{(0)}}{\delta L^{a}} \frac{\delta}{\delta c^{a}}+\frac{\delta \Sigma^{(0)}}{\delta c^{a}} \frac{\delta}{\delta L^{a}}+\frac{\delta \Sigma^{(0)}}{\delta \varrho^{a}} \frac{\delta}{\delta \phi^{a}}+\right. \\
& +\frac{\delta \Sigma^{(0)}}{\delta \phi^{a}} \frac{\delta}{\delta \varrho^{a}}+\frac{\delta \Sigma^{(0)}}{\delta \sigma^{\mu a}} \frac{\delta}{\delta B_{\mu}^{a}}+\frac{\delta \Sigma^{(0)}}{\delta B_{\mu}^{a}} \frac{\delta}{\delta \sigma^{\mu a}}+\frac{\delta \Sigma^{(0)}}{\delta D^{a}} \frac{\delta}{\delta \lambda^{a}}+\frac{\delta \Sigma^{(0)}}{\delta \lambda^{a}} \frac{\delta}{\delta D^{a}}+ \\
& \left.+\frac{\delta \Sigma^{(0)}}{\delta Y^{a}} \frac{\delta}{\delta X^{a}}+\frac{\delta \Sigma^{(0)}}{\delta X^{a}} \frac{\delta}{\delta Y^{a}}+b^{a} \frac{\delta}{\delta \bar{c}^{a}}+d^{a} \frac{\delta}{\delta \bar{\lambda}^{a}}\right]+\chi^{\mu} \frac{\partial}{\partial n^{\mu}} .
\end{aligned}
$$

The algebraic relations (2.20) and (2.21) may be rewritten in terms of functional operators as follows:

$$
\begin{aligned}
\left\{\mathcal{S}_{\Sigma^{(0)}}, \mathcal{S}_{\Sigma^{(0)}}\right\} & =0 \\
\left\{\mathcal{W}_{\mu}, \mathcal{W}_{\nu}\right\} & =0 \\
\{\mathcal{D}, \mathcal{D}\} & =0 \\
\left\{\mathcal{S}_{\Sigma^{(0)}}, \mathcal{D}\right\} & =0 \\
\left\{\mathcal{D}, \mathcal{W}_{\mu}\right\} & =0 \\
\left\{\mathcal{W}_{\mu}, \mathcal{S}_{\Sigma^{(0)}}\right\} & =\mathcal{P}_{\mu},
\end{aligned}
$$

closing off-shell. Here, $\mathcal{P}_{\mu}$ is the Ward operator for translations

$$
\mathcal{P}_{\mu}=\int_{\mathcal{M}} d^{2} x \sum_{\Phi_{i}} \partial_{\mu} \Phi_{i}^{a} \frac{\delta}{\delta \Phi_{i}^{a}}
$$

where $\Phi_{i}^{a}$ stands for all fields.

At the classical level the total action (2.23) is now constrained by the following functional identities:

- Gauge conditions:

$$
\begin{aligned}
\frac{\delta \Sigma^{(0)}}{\delta b^{a}} & =n^{\mu} A_{\mu}^{a} \\
\frac{\delta \Sigma^{(0)}}{\delta d^{a}} & =n^{\mu} B_{\mu}^{a}
\end{aligned}
$$

- Integrated ghost equations:

$$
\begin{aligned}
\mathcal{F}^{a} \Sigma^{(0)} & =\Delta_{\mathcal{F}}^{a}, \\
\mathcal{G}^{a} \Sigma^{(0)} & =\Delta_{\mathcal{G}}^{a},
\end{aligned}
$$


where

$$
\begin{aligned}
\mathcal{F}^{a} & =\int_{\mathcal{M}} d^{2} x\left(\frac{\delta}{\delta c^{a}}-f^{a b c} \bar{c}^{b} \frac{\delta}{\delta b^{c}}-f^{a b c} \bar{\lambda}^{b} \frac{\delta}{\delta d^{c}}\right), \\
\mathcal{G}^{a} & =\int_{\mathcal{M}} d^{2} x\left(\frac{\delta}{\delta \lambda^{a}}-f^{a b c} \bar{\lambda}^{b} \frac{\delta}{\delta b^{c}}\right),
\end{aligned}
$$

and

$$
\begin{aligned}
\Delta_{\mathcal{F}}^{a} & =\int_{\mathcal{M}} d^{2} x f^{a b c}\left(-\varrho^{b} \phi^{c}-Y^{b} X^{c}+L^{b} c^{c}+D^{b} \lambda^{c}-\Omega^{\mu b} A_{\mu}^{c}-\sigma^{\mu b} B_{\mu}^{c}\right) \\
\Delta_{\mathcal{G}}^{a} & =\int_{\mathcal{M}} d^{2} x f^{a b c}\left(D^{b} c^{c}-\varrho^{b} X^{c}-\sigma^{\mu b} A_{\mu}^{c}\right)
\end{aligned}
$$

Note that, once again, the classical breakings $\Delta_{\mathcal{F}}^{a}$ and $\Delta_{\mathcal{G}}^{a}$ are linear in the quantum fields.

- Local antighost equations which are obtained by commuting the gauge conditions with the Slavnov identity:

$$
\begin{aligned}
& \overline{\mathcal{F}}^{a} \Sigma^{(0)}=\left(\frac{\delta}{\delta \bar{c}^{a}}+n^{\mu} \frac{\delta}{\delta \Omega^{\mu a}}\right) \Sigma^{(0)}=0, \\
& \overline{\mathcal{G}}^{a} \Sigma^{(0)}=\left(\frac{\delta}{\delta \bar{\lambda}^{a}}+n^{\mu} \frac{\delta}{\delta \sigma^{\mu a}}\right) \Sigma^{(0)}=0 .
\end{aligned}
$$

- Ward identities of the rigid gauge invariance which are obtained by commuting the ghost equations with the Slavnov identity:

$$
\mathcal{H}^{a} \Sigma^{(0)}=\int_{\mathcal{M}} d^{2} x \sum_{\Phi_{i}} f^{a b c} \Phi_{i}^{b} \frac{\delta \Sigma^{(0)}}{\delta \Phi_{i}^{c}}=0
$$

and

$$
\begin{aligned}
\mathcal{N}^{a} \Sigma^{(0)} & =\int_{\mathcal{M}} d^{2} x f^{a b c}\left(A_{\mu}^{b} \frac{\delta \Sigma^{(0)}}{\delta B_{\mu}^{c}}+\sigma^{\mu b} \frac{\delta \Sigma^{(0)}}{\delta \Omega^{\mu c}}+X^{b} \frac{\delta \Sigma^{(0)}}{\delta \phi^{c}}+\varrho^{b} \frac{\delta \Sigma^{(0)}}{\delta Y^{c}}+\right. \\
& \left.+c^{b} \frac{\delta \Sigma^{(0)}}{\delta \lambda^{c}}+D^{b} \frac{\delta \Sigma^{(0)}}{\delta L^{c}}+\bar{\lambda}^{b} \frac{\delta \Sigma^{(0)}}{\delta \bar{c}^{c}}+d^{b} \frac{\delta \Sigma^{(0)}}{\delta b^{c}}\right)=0
\end{aligned}
$$

In (2.39) $\Phi_{i}$ stands collectively for all fields.

\section{Proof of the finiteness}

This section is devoted to discuss the full symmetry content of the theory at the quantum level, e.g. the question of possible anomalies and the stability problem which ammounts to analyze all invariant counterterms. 
We begin by studying the stability. This requires the analysis of the most general counterterms for the total action and implies to consider the following perturbed action

$$
\Sigma^{\prime}=\Sigma^{(0)}+\Delta
$$

where $\Sigma^{(0)}$ is the total action (2.23) and $\Sigma^{\prime}$ is a functional depending via $\Delta$ on the same fields as $\Sigma^{(0)}$ and satisfying the Slavnov identity (2.24), the Ward identity for the vector supersymmetry (2.26), the two gauge conditions (2.34), the two ghost equations (2.35), the two antighost equations (2.38), the two Ward identities of the rigid gauge invariance (2.39) and (2.40), the Ward identity for the translations (2.33) as well as the Ward identity (2.29). The perturbation $\Delta$ collecting all appropriate invariant counterterms is an intergrated local field polynomial of dimension two and ghost number zero.

Now we are searching for the most general deformation of the classical action such that the perturbed action $\Sigma^{\prime}$ still fullfills the above constraints. The perturbation $\Delta$ must therefore obey the following set of equations:

$$
\begin{aligned}
\frac{\delta \Delta}{\delta b^{a}} & =0, \\
\frac{\delta \Delta}{\delta d^{a}} & =0, \\
\frac{\delta \Delta}{\delta \bar{c}^{a}}+n^{\mu} \frac{\delta \Delta}{\delta \Omega^{\mu a}} & =0, \\
\frac{\delta \Delta}{\delta \bar{\lambda}^{a}}+n^{\mu} \frac{\delta \Delta}{\delta \sigma^{\mu a}} & =0, \\
\mathcal{S}_{\Sigma^{(0)}} & =0, \\
\mathcal{W}_{\mu} \Delta & =0, \\
\mathcal{P}_{\mu} \Delta & =0, \\
\mathcal{D}_{\mathcal{M}} & =0, \\
d^{2} x \frac{\delta \Delta}{\delta c^{a}} & =0, \\
\int_{\mathcal{M}} d^{2} x \frac{\delta \Delta}{\delta \lambda^{a}} & =0, \\
\mathcal{H}^{a} \Delta & =0, \\
\mathcal{N}^{a} \Delta & =0 .
\end{aligned}
$$

The first two equations (3.42) and (3.43) imply that the quantity $\Delta$ does not depend on the multiplier fields $b^{a}$ and $d^{a}$. The validity of (3.44) and (3.45) implies that dependance of $\left(\Omega^{\mu a}, \bar{c}^{a}\right)$ and $\left(\sigma^{\mu a}, \bar{\lambda}^{a}\right)$ is given by the following combinations

$$
\begin{aligned}
& \tilde{\Omega}^{\mu a}=\Omega^{\mu a}-n^{\mu} \bar{c}^{a}, \\
& \tilde{\sigma}^{\mu a}=\sigma^{\mu a}-n^{\mu} \bar{\lambda}^{a} .
\end{aligned}
$$

The equations (3.46)-(3.49), as in reference [11], can be collected into a unified operator $\delta$ :

$$
\delta=\mathcal{S}_{\Sigma^{(0)}}+\xi^{\mu} \mathcal{W}_{\mu}+\varepsilon^{\mu} \mathcal{P}_{\mu}+\eta \mathcal{D}-\int_{\mathcal{M}} d^{2} x \xi^{\mu} \frac{\partial}{\partial \varepsilon^{\mu}}-\int_{\mathcal{M}} d^{2} x \zeta \frac{\partial}{\partial \eta}
$$


producing a single cohomology problem

$$
\delta \Delta=0 .
$$

Here, $\xi^{\mu}$ and $\varepsilon^{\mu}$ are constant vectors of ghost numbers +2 and +1 respectively and the quantities $\zeta$ and $\eta$ are constant scalars of ghost numbers +2 and +1 respectively. It can be easily verified that the operator $\delta$ is nilpotent

$$
\delta^{2}=0 \text {. }
$$

Due to the nilpotency of $\delta$ any expression of the form $\delta \hat{\Delta}$ is automatically a solution of (3.56). A solution of this type is called a trivial solution. Hence, the most general solution of (3.56) reads

$$
\Delta=\Delta_{c}+\delta \hat{\Delta} .
$$

Here, the nontrivial solution $\Delta_{c}$ is $\delta$-closed $\left(\delta \Delta_{c}=0\right)$, but not trivial $\left(\Delta_{c} \neq \delta \hat{\Delta}\right)$. Let us begin with the determination of the nontrivial solution of (3.56). For this purpose we introduce a filtering operator $\mathcal{N}$ :

$$
\mathcal{N}=\int_{\mathcal{M}} d^{2} x \sum_{\Psi} \Psi \frac{\delta}{\delta \Psi},
$$

where $\Psi$ stands for all fields, including $n^{\mu}, \chi^{\mu}, \xi^{\mu}, \varepsilon^{\mu}, \zeta$ and $\eta$. To all fields we assign the homogeneity degree 1 . The filtering operator induces a decomposition of $\delta$ and $\Delta$ according to

$$
\delta=\delta_{0}+\delta_{1}+\ldots, \quad \Delta=\Delta_{1}+\Delta_{2}+\ldots
$$

The operator $\delta_{0}$ does not increase the homogeneity degree while acting on a field polynomial. On the other hand, the operator $\delta_{n}$ increases the homogeneity degree by $n$ units. Similarly, $\Delta_{n}$ is a field polynomial of homogeneity degree $n$. Furthermore, the nilpotency of $\delta$ leads now to

$$
\delta_{0}^{2}=0, \quad\left\{\delta_{0}, \delta_{1}\right\}=0 \quad .
$$

Hence, we obtain from $\delta \Delta=0$ the following relation

$$
\delta_{0} \Delta_{1}=0,
$$

with

$$
\Delta_{1}=\Delta_{c}^{1}+\delta_{0} \hat{\Delta}_{1}
$$

The operator $\delta_{0}$ reads:

$$
\begin{array}{ll}
\delta_{0} A_{\mu}^{a}=\partial_{\mu} c^{a}, & \delta_{0} B_{\mu}^{a}=\partial_{\mu} \lambda^{a}, \\
\delta_{0} \phi^{a}=0 & \delta_{0} X^{a}=0, \\
\delta_{0} c^{a}=0, & \delta_{0} \lambda^{a}=, \\
\delta_{0} L^{a}=\partial_{\mu} \tilde{\Omega}^{\mu a}, & \delta_{0} D^{a}=\partial_{\mu} \tilde{\sigma}^{\mu a}, \\
\delta_{0} \varrho^{a}=\varepsilon^{\mu \nu} \partial_{\mu} A_{\nu}^{a}, & \delta_{0} Y^{a}=\partial_{\mu} A_{\nu}^{a}, \\
\delta_{0} \tilde{\Omega}^{\mu a}=\varepsilon^{\mu \nu} \partial_{\nu} \phi^{a}, & \delta_{0} \tilde{\sigma}^{\mu a}=\varepsilon^{\mu \nu} \partial_{\nu} X^{a}, \\
\delta_{0} n^{\mu}=\chi^{\mu}, & \delta_{0} \chi^{\mu}=0, \\
\delta_{0} \varepsilon^{\mu}=-\xi^{\mu}, & \delta_{0} \xi^{\mu}=0, \\
\delta_{0} \zeta=-\eta, & \delta_{0} \eta=0 .
\end{array}
$$


We notice that the fields $n^{\mu}, \chi^{\mu}, \xi^{\mu}, \varepsilon^{\mu}, \zeta$ and $\eta$ transform under $\delta_{0}$ as doublets, being therefore out of the cohomology [12]. The nontrivial solution $\Delta_{c}^{1}$ can now be written as intergrated local field polynomial of form degree two and ghost number zero:

$$
\Delta_{c}^{1}=\int_{\mathcal{M}} \omega_{2}^{0}
$$

where $\omega_{q}^{p}$ is a field polynomial of form degree $q$ and ghost number $p$. Using the Stoke's theorem, the Poincaré lemma [12] and the relation $\left\{\delta_{0}, d\right\}=0$, where $d$ represents the nilpotent exterior derivative $\left(d^{2}=0\right)$, we obtain the following tower of descent equations:

$$
\begin{aligned}
\delta_{0} \omega_{2}^{0}+d \omega_{1}^{1} & =0 \\
\delta_{0} \omega_{1}^{1}+d \omega_{0}^{2} & =0 \\
\delta_{0} \omega_{0}^{2} & =0
\end{aligned} .
$$

The tower of descent equations (3.66) has been solved in [13], where it was shown that the ghost equations (3.50) and (3.51) imply that the solution $\Delta_{c}^{1}$ must vanish identically. The usefulness of the decomposition (3.60) relies on a very general theorem stating that the cohomology of the complete operator $\delta$ is isomorphic to a subspace of the cohomology of the operator $\delta_{0}$.

Next, we move to the computation of the trivial counterterms. These are constrained by the dimension and ghost number requirements. The scalar fields $\phi$ and $X$ both have vanishing dimension and ghost number zero, so that an arbitrary combination of them may appear infinitely many times in the counterterm. For the most general and possible combination of these fields we use the notation $f^{\alpha}[\phi, X]$ as introduced in [13]:

$$
f^{\alpha}[\phi, X]=\sum_{\left\{n_{i}\right\},\left\{m_{i}\right\}=0}^{\infty} \beta_{n_{i}, m_{i}}^{\alpha}\left(\prod_{i=0}^{\infty} \phi^{n_{i}} X^{m_{i}}\right)
$$

where $\left\{n_{i}\right\}$ and $\left\{m_{i}\right\}$ are understood as $\left\{n_{0}, n_{1}, \ldots\right\}$ and $\left\{m_{0}, m_{1}, \ldots\right\}$, respectively. Here, $\beta_{n_{i}, m_{i}}^{\alpha}$ are constant coefficients to be determined. The most general trivial counterterm $\delta \hat{\Delta}$ where $\hat{\Delta}$ has dimension 2 and carries ghost number -1 reads:

$$
\begin{aligned}
\delta \hat{\Delta} & =\delta \int_{\mathcal{M}} d^{2} x \operatorname{Tr}\left(\varrho f^{1}+Y f^{2}+\tilde{\Omega}^{\nu} f^{3} A_{\nu} f^{4}+\varepsilon_{\mu \nu} \tilde{\Omega}^{\mu} f^{5} A^{\nu} f^{6}+n_{\mu} n_{\nu} \tilde{\Omega}^{\mu} f^{7} A^{\nu} f^{8}+\right. \\
& +\varepsilon^{\mu \nu} n_{\mu} \tilde{\Omega}_{\nu} f^{9} n^{\rho} A_{\rho} f^{10}+n^{\mu} \tilde{\Omega}_{\mu} f^{11} \varepsilon^{\nu \rho} n_{\nu} A_{\rho} f^{12}+\varepsilon^{\mu \nu} n_{\mu} \tilde{\Omega}_{\nu} f^{13} \varepsilon^{\rho \sigma} n_{\rho} A_{\sigma} f^{14}+ \\
& +\tilde{\sigma}^{\nu} f^{15} A_{\nu} f^{16}+\varepsilon_{\mu \nu} \tilde{\sigma}^{\mu} f^{17} A^{\nu} f^{18}+n_{\mu} n_{\nu} \tilde{\sigma}^{\mu} f^{19} A^{\nu} f^{20}+ \\
& +n^{\mu} \tilde{\sigma}_{\mu} f^{23} \varepsilon^{\nu \rho} n_{\nu} A_{\rho} f^{24}+\varepsilon^{\mu \nu} n_{\mu} \tilde{\sigma}_{\nu} f^{25} \varepsilon^{\rho \sigma} n_{\rho} A_{\sigma} f^{26}+\tilde{\Omega}^{\nu} f^{27} B_{\nu} f^{28}+\varepsilon_{\mu \nu} \tilde{\Omega}^{\mu} f^{29} B^{\nu} f^{30}+ \\
& +n_{\mu} n_{\nu} \tilde{\Omega}^{\mu} f^{31} B^{\nu} f^{32}+\varepsilon^{\mu \nu} n_{\mu} \tilde{\Omega}_{\nu} f^{33} n^{\rho} B_{\rho} f^{34}+n^{\mu} \tilde{\Omega}_{\mu} f^{35} \varepsilon^{\nu \rho} n_{\nu} B_{\rho} f^{36}+ \\
& +\varepsilon^{\mu \nu} n_{\mu} \tilde{\Omega}_{\nu} f^{37} \varepsilon^{\rho \sigma} n_{\rho} B_{\sigma} f^{38}+\tilde{\sigma}^{\nu} f^{39} B_{\nu} f^{40}+\varepsilon_{\mu \nu} \tilde{\sigma}^{\mu} f^{41} B^{\nu} f^{42}+n_{\mu} n_{\nu} \tilde{\sigma}^{\mu} f^{43} B^{\nu} f^{44}+ \\
& +\varepsilon^{\mu \nu} n_{\mu} \tilde{\sigma}_{\nu} f^{45} n^{\rho} B_{\rho} f^{46}+n^{\mu} \tilde{\sigma}_{\mu} f^{47} \varepsilon^{\nu \rho} n_{\nu} B_{\rho} f^{48}+\varepsilon^{\mu \nu} n_{\mu} \tilde{\sigma}_{\nu} f^{49} \varepsilon^{\rho \sigma} n_{\rho} B_{\sigma} f^{50}+ \\
& +\left(\partial^{\nu} \tilde{\Omega}_{\nu}\right) f^{51}+\varepsilon_{\mu \nu}\left(\partial^{\mu} \tilde{\Omega}^{\nu}\right) f^{52}+n_{\mu} n_{\nu} \partial^{\mu} \tilde{\Omega}^{\nu} f^{53}+\varepsilon^{\mu \nu} n_{\mu} \partial_{\nu}\left(n^{\rho} \tilde{\Omega}_{\rho}\right) f^{54}+ \\
& +n^{\mu} \partial_{\mu}\left(\varepsilon^{\nu \rho} n_{\nu} \tilde{\Omega}_{\rho}\right) f^{55}+\varepsilon^{\mu \nu} n_{\mu} \partial_{\nu}\left(\varepsilon^{\rho \sigma} n_{\rho} \tilde{\Omega}_{\sigma}\right) f^{56}+\left(\partial^{\nu} \tilde{\sigma}_{\nu}\right) f^{57}+\varepsilon_{\mu \nu}\left(\partial^{\mu} \tilde{\sigma}^{\nu}\right) f^{58}+
\end{aligned}
$$




$$
\begin{aligned}
& +n_{\mu} n_{\nu} \partial^{\mu} \tilde{\sigma}^{\nu} f^{59}+\varepsilon^{\mu \nu} n_{\mu} \partial_{\nu}\left(n^{\rho} \tilde{\sigma}_{\rho}\right) f^{60}+n^{\mu} \partial_{\mu}\left(\varepsilon^{\nu \rho} n_{\nu} \tilde{\sigma}_{\rho}\right) f^{61}+\varepsilon^{\mu \nu} n_{\mu} \partial_{\nu}\left(\varepsilon^{\rho \sigma} n_{\rho} \tilde{\sigma}_{\sigma}\right) f^{62}+ \\
& +L f^{63} c f^{64}+\Lambda f^{65} c f^{66}+L f^{67} \lambda f^{68}+\Lambda f^{69} \lambda f^{70}+n^{\mu} \chi_{\mu} L f^{71}+\varepsilon^{\mu \nu} n_{\mu} \chi_{\nu} L f^{72}+ \\
& +n^{\mu} \chi_{\mu} \Lambda f^{73}+\varepsilon^{\mu \nu} n_{\mu} \chi_{\nu} \Lambda f^{74}+n^{\mu} \chi_{\mu} \tilde{\sigma}^{\nu} \tilde{\sigma}_{\nu} f^{75}+n^{\mu} \chi_{\mu} \tilde{\sigma}^{\nu} f^{76} \tilde{\Omega}_{\nu} f^{77}+n^{\mu} \chi_{\mu} \tilde{\Omega}^{\nu} \tilde{\Omega}_{\nu} f^{78}+ \\
& +n^{\mu} \tilde{\Omega}_{\mu} \chi^{\nu} f^{79} \tilde{\sigma}_{\nu} f^{80}+n^{\mu} \tilde{\sigma}_{\mu} f^{81} \tilde{\Omega}^{\nu} \chi_{\nu} f^{82}+n^{\mu} \chi^{\nu} \tilde{\sigma}_{\mu} f^{83} \tilde{\sigma}_{\nu} f^{84}+n^{\mu} \chi^{\nu} \tilde{\Omega}_{\mu} f^{85} \tilde{\Omega}_{\nu} f^{86}+ \\
& +n^{\mu} \chi_{\mu} n^{\nu} \tilde{\sigma}_{\nu} n^{\rho} \tilde{\sigma}_{\rho} f^{87}+n^{\mu} \chi_{\mu} n^{\nu} \tilde{\sigma}_{\nu} f^{88} n^{\rho} \tilde{\Omega}_{\rho} f^{89}+n^{\mu} \chi_{\mu} n^{\nu} \tilde{\Omega}_{\nu} n^{\rho} \tilde{\Omega}_{\rho} f^{90}+ \\
& +n^{\mu} \chi_{\mu} \varepsilon^{\nu \rho} \tilde{\sigma}_{\nu} \tilde{\sigma}_{\rho} f^{91}+n^{\mu} \chi_{\mu} \varepsilon^{\nu \rho} \tilde{\sigma}_{\nu} f^{92} \tilde{\Omega}_{\rho} f^{93}+n^{\mu} \chi_{\mu} \varepsilon^{\nu \rho} \tilde{\Omega}_{\nu} \tilde{\Omega}_{\rho} f^{94}+ \\
& +n^{\mu} \tilde{\sigma}_{\mu} f^{95} \varepsilon^{\nu \rho} \chi_{\nu} \tilde{\sigma}_{\rho} f^{96}+n^{\mu} \tilde{\sigma}_{\mu} f^{97} \varepsilon^{\nu \rho} \tilde{\Omega}^{\nu} \chi_{\rho} f^{98}+n^{\mu} \tilde{\Omega}_{\mu} f^{99} \varepsilon^{\nu \rho} \chi_{\nu} \tilde{\sigma}_{\rho} f^{100} \\
& +n^{\mu} \tilde{\Omega}_{\mu} f^{101} \varepsilon^{\nu \rho} \chi_{\nu} \tilde{\Omega}_{\rho} f^{102}+\varepsilon^{\mu \nu} n_{\mu} \chi_{\nu} \tilde{\sigma}^{\rho} \tilde{\sigma}_{\rho} f^{103}+\varepsilon^{\mu \nu} n_{\mu} \chi_{\nu} \tilde{\sigma}^{\rho} f^{104} \tilde{\Omega}_{\rho} f^{105}+ \\
& +\varepsilon^{\mu \nu} n_{\mu} \chi_{\nu} \tilde{\Omega}^{\rho} \tilde{\Omega}_{\rho} f^{106}+\varepsilon^{\mu \nu} n_{\mu} \tilde{\sigma}_{\nu} f^{107} \chi^{\rho} \tilde{\sigma}_{\rho} f^{108}+\varepsilon^{\mu \nu} n_{\mu} \tilde{\Omega}_{\nu} f^{109} \chi^{\rho} \tilde{\sigma}_{\rho} f^{110}+ \\
& +\varepsilon^{\mu \nu} n_{\mu} \tilde{\Omega}_{\nu} f^{111} \chi^{\rho} \tilde{\Omega}_{\rho} f^{112}+\varepsilon^{\mu \nu} n_{\mu} \chi_{\nu} \varepsilon^{\rho \sigma} \tilde{\sigma}_{\rho} \tilde{\sigma}_{\sigma} f^{113}+\varepsilon^{\mu \nu} n_{\mu} \chi_{\nu} \varepsilon^{\rho \sigma} \tilde{\sigma}_{\rho} f^{114} \tilde{\Omega}_{\sigma} f^{115}+ \\
& +\varepsilon^{\mu \nu} n_{\mu} \chi_{\nu} \varepsilon^{\rho \sigma} \tilde{\Omega}_{\rho} \tilde{\Omega}_{\sigma} f^{116}+\varepsilon^{\mu \nu} n_{\mu} \tilde{\sigma}_{\nu} f^{117} \varepsilon^{\rho \sigma} \chi_{\rho} \tilde{\sigma}_{\sigma} f^{118}+\varepsilon^{\mu \nu} n_{\mu} \tilde{\sigma}_{\nu} f^{119} \varepsilon^{\rho \sigma} \chi_{\rho} \tilde{\Omega}_{\sigma} f^{120}+ \\
& +\varepsilon^{\mu \nu} n_{\mu} \tilde{\Omega}_{\nu} f^{121} \varepsilon^{\rho \sigma} \chi_{\rho} \tilde{\sigma}_{\sigma} f^{122}+\varepsilon^{\mu \nu} n_{\mu} \tilde{\Omega}_{\nu} f^{123} \varepsilon^{\rho \sigma} \chi_{\rho} \tilde{\Omega}_{\sigma} f^{124}+ \\
& +\varepsilon^{\mu \nu} n_{\mu} \chi_{\nu} \varepsilon^{\rho \sigma} n_{\rho} \tilde{\sigma}_{\sigma} f^{125} \varepsilon^{\omega \tau} n_{\omega} \tilde{\sigma}_{\tau} f^{126}+\varepsilon^{\mu \nu} n_{\mu} \chi_{\mu} \varepsilon^{\rho \sigma} n_{\rho} \tilde{\sigma}_{\sigma} f^{127} \varepsilon^{\omega \tau} n_{\omega} \tilde{\Omega}_{\tau} f^{128}+ \\
& +\varepsilon^{\mu \nu} n_{\mu} \chi_{\mu} \varepsilon^{\rho \sigma} n_{\rho} \tilde{\Omega}_{\sigma} f^{129} \varepsilon^{\omega \tau} n_{\omega} \tilde{\Omega}_{\tau} f^{130}+\varepsilon^{\mu \nu} n_{\mu} \chi_{\nu} \varepsilon^{\rho \sigma} n_{\rho} \tilde{\sigma}_{\sigma} f^{131} n^{\omega} \tilde{\sigma}_{\omega} f^{132}+ \\
& \left.+\varepsilon^{\mu \nu} n_{\mu} \chi_{\nu} \varepsilon^{\rho \sigma} n_{\rho} \tilde{\sigma}_{\sigma} f^{133} n^{\omega} \tilde{\Omega}_{\omega} f^{134}+\varepsilon^{\mu \nu} n_{\mu} \chi_{\nu} \varepsilon^{\rho \sigma} n_{\rho} \tilde{\Omega}_{\sigma} f^{135} n^{\omega} \tilde{\Omega}_{\omega} f^{137}\right) \text {. }
\end{aligned}
$$

The trivial counterterm may depend on the quantities $\xi^{\mu}, \varepsilon^{\mu}, \zeta$ and $\eta$ which do not appear in the total action (2.23). For this reason we demand the expression (3.68) to be invariant under the Ward-operators of the vector supersymmetry and translations as well as under the Ward-operator $\mathcal{D}$. A lengthy and tedious analysis yields that the counterterm satisfying these conditions must vanish identically, so that for the determination of the trivial counterterms contrary to [13] the use of the ghost equations (3.50) and (3.51) is not needed any more due to the invariance under the symmetry $\mathcal{D}$.

The last problem is devoted to the discussion of possible existance of breaking of the symmetries. As shown in reference [13] for Landau gauge 9] and under the assumption that the quantum action principle is also valid in the case of noncovariant gauges 15, the symmetries appearing in the model do not admit any anomalies and are valid at the full quantum level. This completes the proof of finiteness of the model to all orders of perturbation theory.

\section{References}

[1] G. T. Horowitz and M. Srednicki, Comm. Math. Phys. 130 (1990) 83;

G. T. Horowitz, Comm. Math. Phys. 125 (1989) 417;

[2] S. K. Donaldson, J. Diff. Geom. 18 (1983) 279;

Ann. Phys. (N.Y.) 205 (1991) 130; Topology 29 (1990) 257;

[3] D. Birmingham, M. Blau, M. Rakowski and G. Thompson, Phys. Rep. 209 (1991) 129 
[4] E. Witten, Commun. Math. Phys. 121 (1989) 351;

[5] A. H. Chamseddine and D. Wyler, Nucl. Phys. B340 (1990) 595; Phys. Lett. B228 (595);

[6] A. Blasi and N. Maggiore, Class. Quantum Grav. 10 (1993) 37;

[7] S. Emery, M. Krüger, J. Rant, M. Schweda and T. Sommer, Il Nuovo Cimento, Vol. $111 \mathrm{~A}$, N. 11;

[8] C. M. Becchi, A. Rouet, R. Stora, Comm. Math. Phys. 42 (1975) 127; Ann. Phys. (N.Y.) 98 (1976) 287;

[9] O. Piguet and S. P. Sorella, Algebraic Renormalization, Perturbative Renormalization, Symmetries and Anomalies, Lecture Notes in Physics, New Series m: 28, Springer Verlag 1995;

[10] O. Piguet and K. Sibold, Nucl. Phys. B253 (1985) 517;

[11] C. M. Becchi, A. Blasi, G. Bonneau, R. Collina and F. Delduc, Comm. Math. Phys.120 (1988);

[12] F. Brandt, N. Dragon, M. Kreuzer, Phys. Lett. B231 (1989) 263; Nucl. Phys. B332 (1990) 224; Nucl. Phys. B340 (1990) 187;

[13] R. Leitgeb, M. Schweda and H. Zerrouki, Nucl. Phys. B542 (1999) 425;

[14] A. Brandhuber, S. Emery, K. Landsteiner and M. Schweda, Helv. Phys. Acta 66 (1995) 126-138;

[15] A. Boresch, S. Emery, O. Moritsch, M. Schweda, T. Sommer and H. Zerrouki, Applications of noncovariant gauges in the algebraic renormalization procedure, World Scientific 1998; 